\title{
PRIORITIZATION OF STRATEGIC GUIDELINES AS PART OF THE STRATEGIC PLAN 2010-2014 FOR A VENEZUELAN UNIVERSITY USING AHP
}

\author{
Aidaelena Smith-Perera* \\ Universidad Metropolitana \\ Caracas, Venezuela \\ E-mail: asmith@unimet.edu.ve \\ Xavier Figarella \\ Universidad Metropolitana \\ Caracas, Venezuela \\ E-mail: xfigarella@unimet.edu.ve
}

\begin{abstract}
Definition and execution of a Strategic Plan for a Venezuelan University; as Critical Success Factor in this Plan, it will be necessary to count on stakeholders involvement (professors, students, employees, organizations, among others) in the decision making process. Venezuelan universities are submerged in a changing country; in this sense, they must be always prepared for adapting to new challenges and to adjust and continue to be able to generate knowledge valuable for Venezuelan society. As part of the redefinition of the 2010-2014 strategic plan, Universidad Metropolitana defined six strategic guidelines or key development areas that are aligned with University's mission and vision. The strategic guidelines are the base for projects to be developed for years to come. Once strategic guidelines were openly defined, discussed and accepted there was a need to prioritize them. Based on the experience using AHP and ANP, the Planning Commission decided to utilize AHP as a technique to determine relative importance to each one of the strategic guidelines objectively and with the participation of major stakeholders in one session. The 24 Participants were divided into five groups. Each stakeholder answered a questionnaire of 15 questions with his/her judgments. Results are divided into groups and a combined for all groups. Results were presented to participants and satisfaction was measured. Calculated priorities are currently the based to annually define projects.
\end{abstract}

Keywords: Strategic Plan, Strategic guidelines, key development area, AHP, Unimet.

\section{Introduction}

Universidad Metropolitana (Unimet) is a private university of a 5500 students both undergraduate and graduate studies, located in Caracas, Venezuela. It offers 15 undergraduate programs and more than 35 graduate programs. Graduate programs include diplomas, masters and specialties. The current strategic planning process started in January 2010, based on two previous plans. According to definitions given by Hunt et al (1997), Unimet is a private non-profit educational institution which incomes come from tuition, contributions and auxiliary goods and services.

This ongoing process has been different since all planning process has been made in house, with no support from external consultants.

\footnotetext{
${ }^{*}$ Corresponding author
} 
According to Hunt et al (1997) "Planning as part of the management process is crucial to the success of the institution. This is especially true for private colleges, universities, and graduate schools". Hunt et al (1997) also discussed that with increasing volatility of the environment, organizations have moved to strategic planning in order to maintain a proper balance between the organization and the demands of the external environment and internal culture.

Having the experience of a previous two strategic plans in Unimet board of director had been committed to go ahead with strategic planning to incorporate some of the innovative areas already in use in the university, such as entrepreneurship, university social responsibility, among other. It has been the intension, moreover to develop the know-how in strategic planning inside Unimet, which will guarantee that follow-up is more effective. Etzkowits et al (2000) proposes among other authors propose the evaluation of universities to an enhanced role in technological innovateion in order to contribute to economics, industry and government.

All Unimet government agreed on the importance in going ahead with a strategic planning using Unimet own personnel. They also agreed that having the planning process done by ourselves was a win-win decision. However, the use of AHP had not been tested before and there is little knowledge internally on the strengths of it as a multicriteria decision technique. Even though the author has had experience on AHP, she has never applied it to obtain results or use these results.

\section{Strategic Planning Process at Universidad Metropolitana}

The board of directors is the higher authority in Unimet, as shown in figure 1 (www.unimet.edu.ve). It is integrated by the president, the rector and 24 more members according to normative. The board has the responsibility of the general direction. Rector is the highest academic and administrative authority within Unimet. Its responsibilities are determined by the normative and regulations given by the board of directors. Meanwhile academic vice rector is in charge of coordinating academic, culture, scientific and technological activities, while administrative vice rector directs and coordinates administrative actions along with the rector.

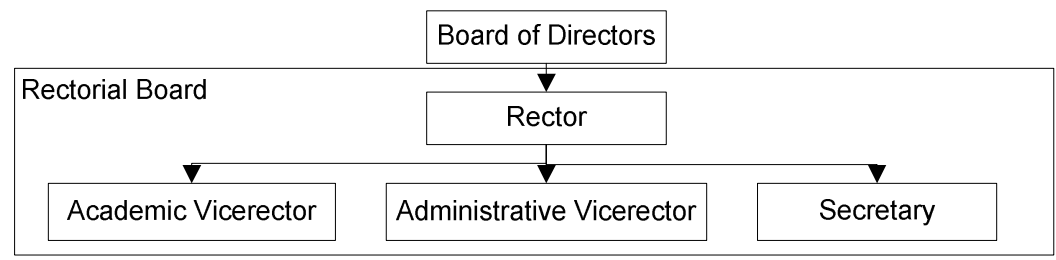

Figure 1. Unimet government organization chart

Current strategic planning process in Unimet initiated in 2010 when Planning Commission was designated by university rectorial board by the authority given by board of directors with the mandate to prepare strategic plan for years 2010-2014. Planning Commission was chaired by Unimet's academic vice rector at the time. Planning Commission is supported by a technical committee. Planning Commission is integrated by 18 people: one professor for each faculty, one for each union, association and organization within Unimet and one for different levels of university government. Technical committee included by eight people supporting the commission. 


\section{A. Smith-Perera/ Prioritization of strategic guidelines as part of the Strategic plan 2010-2014 for a venezuelan university using ahp}

Strategic planning process in Unimet, as shown in figure 2, is divided into 3 stages and is, by definition, an ongoing process with constant revision and adaptation. At current time, project definition in stage 3 is taking place in each administrative unit in Unimet. Stage 1 which in part is described in this investigation, started in January 2010 using pre-existing information such as mission, vision and 11 pre-defined strategic guidelines, which then led to defining six strategic guidelines o key development areas.

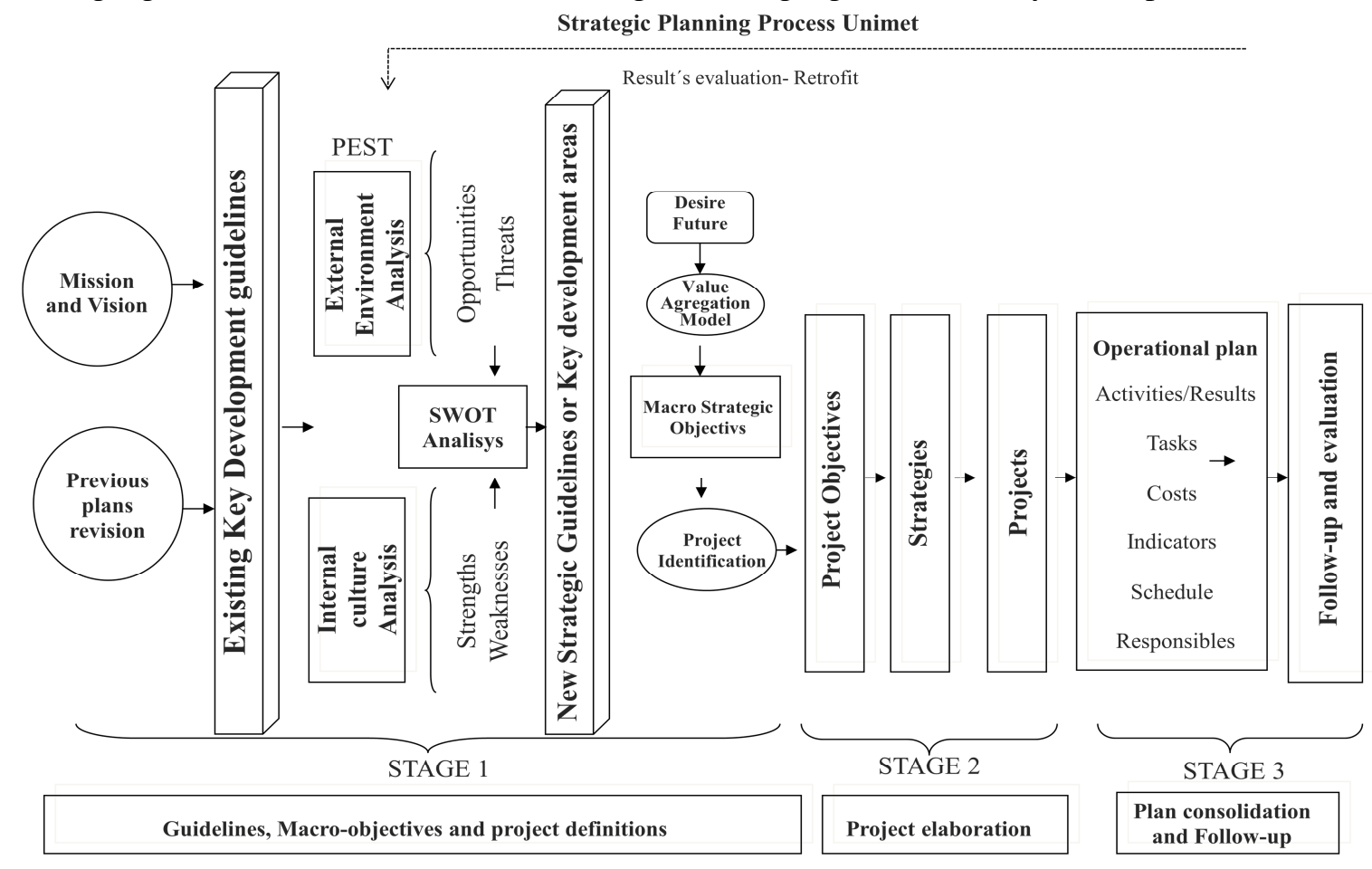

Figure 2. Strategic Planning Process in Unimet by technical committee

For more than a year planning commission had weekly meetings to revise and redefine Strategic Guidelines or key development areas (KDA), macro strategic objectives and project objectives related to guidelines. At the end of this process six KDA were defined.

The six strategic guidelines or KDA are:

1. Strengthen as a vanguard university (L1)

2. University with recognized academic activity (L2)

3. Entrepreneurial university (L3)

4. Financial balance and assurance of viability (L4)

5. Functionality of university governance (L5)

6. University Social Responsibility (L6)

Once the six KDA were discussed and approved by the Planning Commission they were presented for approval to board of directors and once approved they were broadcasted and presented to internal community in order to make the community participant. At this point there was only a definition of key development areas, objectives and macro projects but there was a lack of relative importance or weights to KDA. We will discussed the methodology proposed and applied to assigning weights to KDA assigned 
as criteria to the multicriteria decision problem of establishing and executing projects in Unimet for the years to come.

\section{MCDA applied to Strategic Planning}

Stewart et al (2013) explore the integration of MCDA and scenario planning as part of strategic planning process.

Meanwhile, Arbel and Orgler (1990) describe a methodology based on AHP to evaluate bank mergers and acquisitions strategy and performed a test with participation of a board of directors of a billion dollar bank holding company and concluded that comparing to existing techniques for same problem, AHP provides a "useful, simple and powerful tool for dealing with strategic planning in banking".

In different industries, Oeltjenbruns et al (1995) propose the use of AHP to evaluate different planning alternatives to a manufacturing decision making of deciding what to do with machines, having different alternatives and conflicting criteria.

\section{Methodology established to prioritize strategic guidelines}

At this point, planning commission proposes the technical committee to find a procedure to assign importance by weighting the six KDA understanding that the procedure had to produce results the same day during a visualization activity that was going to take place with the participation of rectorial board (University rector, academic vice rector, administrative vice rector and general secretary), deans and representatives from internal organizations, unions and associations

An AHP based procedure was proposed, evaluated and tested with members of technical committee. The proposed procedure was the following:

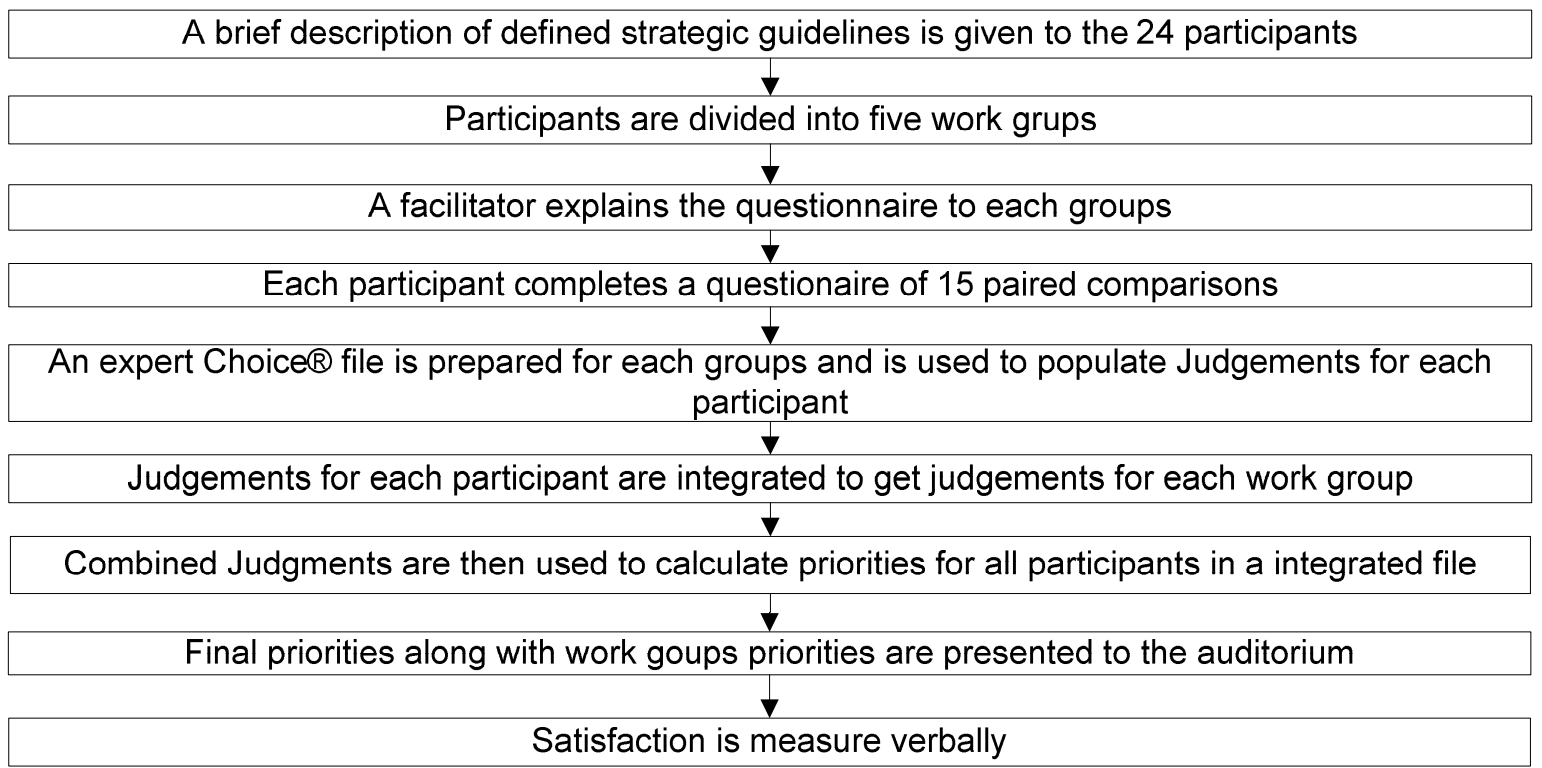

Figure 3: AHP-based procedure to prioritize strategic guidelines in Unimet 


\section{A. Smith-Perera/ Prioritization of strategic guidelines as part of the Strategic plan 2010-2014 for a venezuelan university using ahp}

Due to limitations in time and the importance of the participants, it is not possible to go back and revise judgments with participants to improve inconsistencies, if they are higher than recommended. However, it is possible to take decisions when calculating and decide whether or not inconsistent participants are going to be included when integrating results. Satisfaction cannot be measure using a questionnaire due to lack of time. Participants will have time during plenary to express their impressions of the procedure.

Having the results with no time to review, correct and revise judgments is the most delicate part when implementing this procedure, understanding that results are going to be accepted as valid and will be presented to the board as calculated originally.

The goal of the AHP problem presented to experts was: "The University we dream". The six KDA are presented as criteria for the goal to achieve. The problem structure is shown on figure 4.

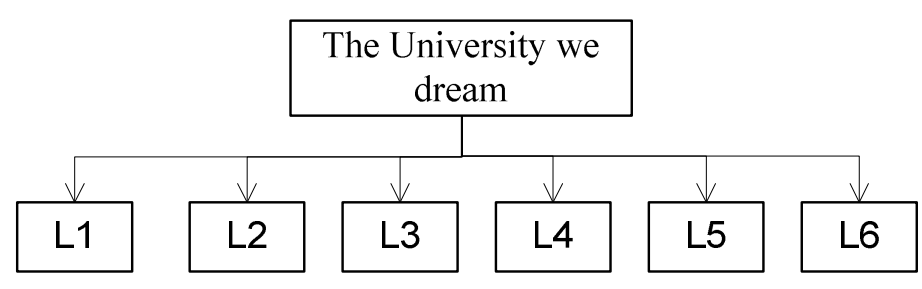

Figure 4. Unimet Model for prioritizing KDA

The questionnaire has the following structure:

Compare the following strategic guidelines according its influence to the achievement of the desired university by medium term

A: L1: Strengthen as a vanguard university

B: L2: University with recognized academic activity

\begin{tabular}{|l|l|l|l|l|}
\hline ¿Most influent? & $\square$ A & $\square$ B & $\square$ Same & \\
\hline ¿How much more? $\square$ Few & $\square$ Moderate & $\square$ Strong & $\square$ Extreme \\
\hline
\end{tabular}

It was important in the questionnaire to keep the name on the KDA in each question in order to keep experts focused on the question.

Combination of judgments to obtain final KDA priorities was done in two steps as shown in figure 5.

\subsection{Visualization activity}

The visualization activity was summoned for July $19^{\text {th }}, 2011$ and a total of 24 participants that were divided into five work groups. The agenda included complete group activities, five presentations and wok group activities when questionnaire completion took place. Questionnaire completion was done in 20 minutes and participants judgments were used to populate files for each work group during hours of visualization activity. Calculations were performed and result judgments for each work group populated the last file for the whole group of participants.

At plenary, participants give feedback on this procedure. Satisfaction was complete and no complains were expressed. 


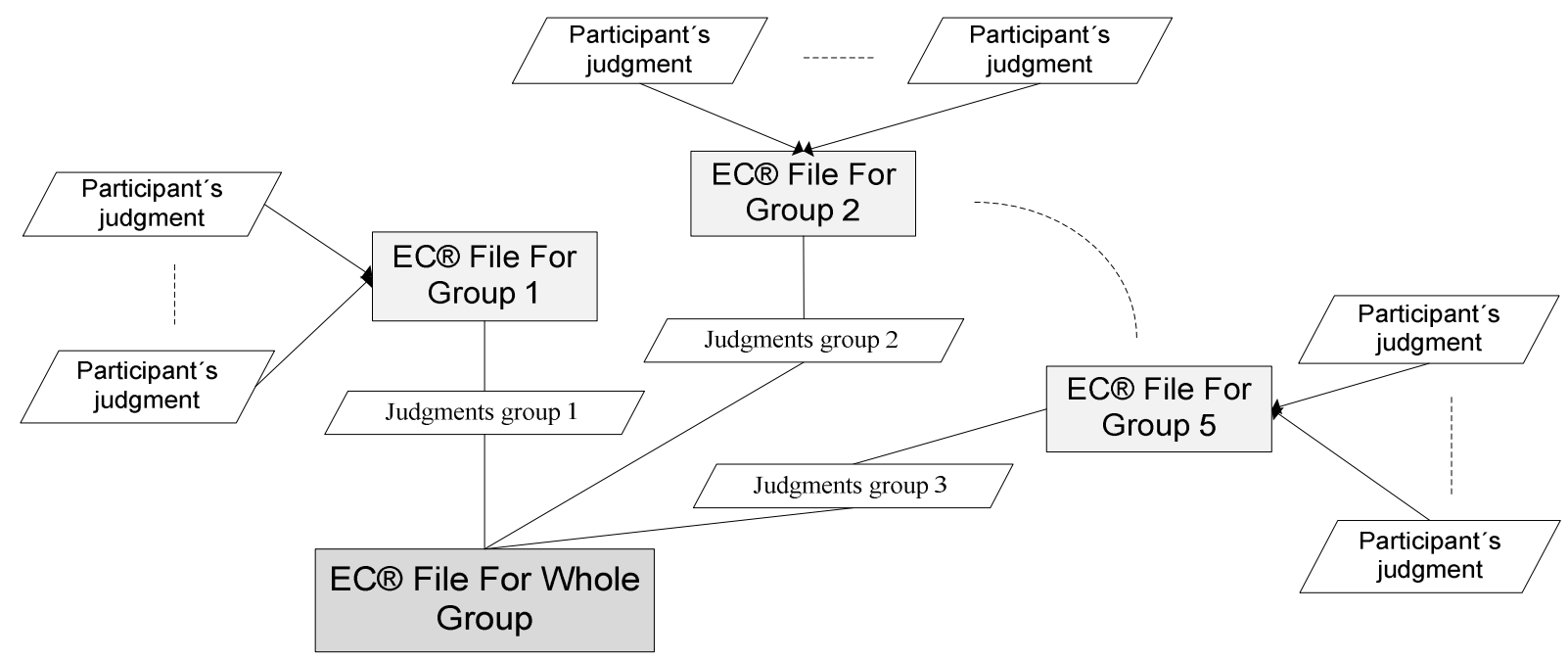

Figure 5. Steps to combine judgments to obtain priorities

\section{Results}

Even though all participants were experts and part of the decision making and knew the implications of assigning weights to key development areas for the years to come, the starting point of the definition of the activity plans for their area, inconsistencies were very high for some participants. Due to the nature of the activity and the time frame available for each participant, revision of inconsistencies with each one of the participants was not possible. It was mandatory to get results that same day. Resulting priorities for each group are shown in tables. The Planning Commission decided to calculate using judgments for all participants, except one that had an emergency and was not available to fulfill the questionnaire when planned according to the agenda. Table 1 show priorities calculated with judgments from all participants except for one that was not available at the time of completing the questionnaire (Original) and priorities adopted by planning commission and being used currently (In use).

Table 1.Priorities calculated with all participants and in used currently ordered from higher to lower are:

\begin{tabular}{|l|l|l|}
\hline KDA & Original (\%) & In Use (\%) \\
\hline L4 & 24.7 & 25 \\
\hline L2 & 21.8 & 22 \\
\hline L6 & 17.1 & 17 \\
\hline L5 & 13.1 & 13 \\
\hline L1 & 12.4 & 12 \\
\hline L3 & 11.0 & 11 \\
\hline
\end{tabular}

As shown on table 2, inconsistencies were as high as $128 \%$. If inconsistency is taken as recommended (10\%) only 5 out of 23 experts would be taken into account, so, a higher acceptable inconsistency was selected to get a higher number of participants when combining judgments. However, even though an acceptable inconsistency limit was established, group five would have kept only judgments from one expert. 

university using ahp

Table 2 .Inconsistencies for experts in each group

\begin{tabular}{|c|c|c|c|c|c|}
\hline Exp & Group 1 & Group 2 & Group 3 & Group 4 & Group 5 \\
\hline 1 & $128 \%$ & $43 \%$ & $11 \%$ & N/A & $34 \%$ \\
\hline 2 & $12 \%$ & $8 \%$ & $84 \%$ & $17 \%$ & $44 \%$ \\
\hline 3 & $7 \%$ & $25 \%$ & $17 \%$ & $16 \%$ & $18 \%$ \\
\hline 4 & $5 \%$ & $6 \%$ & $9 \%$ & $25 \%$ & $24 \%$ \\
\hline 5 & $22 \%$ & $19 \%$ & & & $32 \%$ \\
\hline 6 & & $80 \%$ & & & \\
\hline
\end{tabular}

Having inconsistencies shown on table 2, new priorities were calculated eliminating judgments for experts whose inconsistencies were higher than 20\%. Nine experts' judgments were eliminated from calculation and integration of judgments was done using the same procedure as in original calculations. Five files (one for each group) and then combined judgments were used for a final file as a group's judgments. Results are shown on table 3, along with original priorities. Meanwhile, figure 4 shows the difference graphically.

Table 3 .New priorities and differences

\begin{tabular}{|l|l|l|l|}
\hline KDA & Original (\%) & New (\%) & Difference (\%) \\
\hline L4 & 24.7 & 22.8 & -7.69 \\
\hline L2 & 21.8 & 27.2 & 24.77 \\
\hline L6 & 17.1 & 17.2 & 0.58 \\
\hline L5 & 13.1 & 9.9 & -24.43 \\
\hline L1 & 12.4 & 12.7 & 2.42 \\
\hline L3 & 11.0 & 10.2 & -7.27 \\
\hline
\end{tabular}

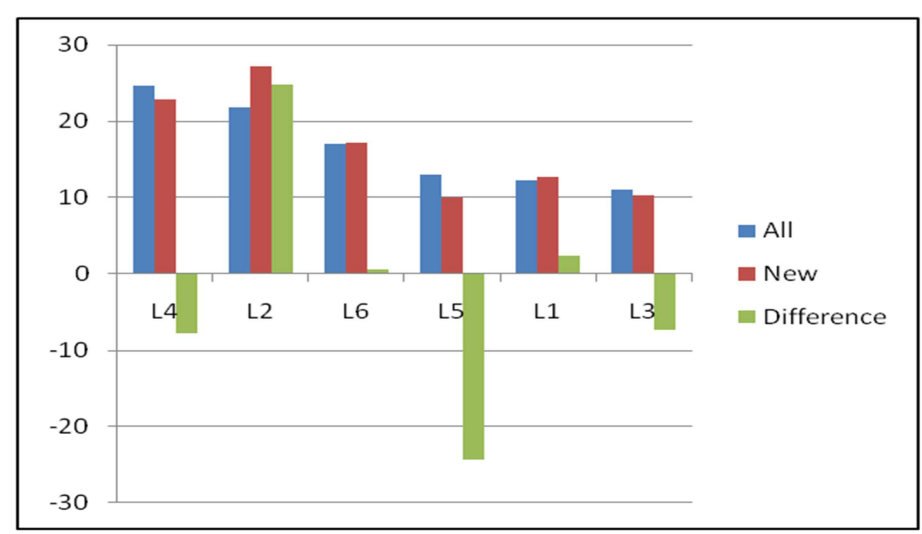

Figure 4 .Comparison Original and new priorities

The new results are under revision to determine the implications on changing in-use priorities for the KDA by Planning Commission. 
Satisfaction was measure in the plenary session when results were presented and no comments were relevant on

\section{Conclusions}

AHP is a valid decision making technique to get preferences, combining them and assigned priorities when interests are diverse and experts have different backgrounds and expectations. In this investigation all experts did not know the technique or the questionnaires from before and were not aware of the procedure to be followed in advance, even so, results were adequate and participants were satisfied. However, having to assign priorities to such an important area as the KDA cannot be limited to few hours

However when results need to be calculated with a limit time frame, a very careful look at inconsistencies need to be taken care of. In such a group of experts like the one in this investigation it is very delicate to eliminate judgments from experts whose inconsistencies are high, since participation and the sensation of being a decision making is important. In Venezuela it can quite delicate to go back to an expert to revise and substitute judgments to get better inconsistencies. There is still much to do in Unimet in this subject.

When combining judgments from all experts in each group, even those whose inconsistencies were higher than $20 \%$, the group inconsistency always resulted below $10 \%$.

\section{REFERENCES}

Arbel, A., Orgler, Y.E. (1990). An application of the AHP to bank strategic planning: The mergers and acquisitions. European Journal of Operational Research, 48, 27-37.

Etzkowitz, H., Webster, A.,Gebhardt, C., Cantizano, B. (2000). The future of the university and the university of the future: evolution of ivory tower to entrepreneurial paradigm. Research Policy, 29, 313330.

Hunt, C., Oosting, K.W., Stevens, R.,Loudon, D., Migliore, R.H. (1997). Strategic planning for private higher education. Binghamton, NY: The Haworth Press, Inc.

Oeltjenbruns, H., Kolarik, W.,Schnadt-Kirschner, R. (1995). Strategic planning in manufacturing sistemas - AHP application to an equipment replacement decision. Internationa Journal Production Economics, 38, 189-197.

Stewart, T.J., French, S., Rios, J. (2013). Integrating multicriteria decision analysis and scenario planning—Review and extension. Omega, 41(4), 679-68. 